\title{
REDES SOCIALES Y DESARROLLO HUMANO
}

SOCIAL NETWORKS AND HUMAN DEVELOPMENT

Sara Gallego Trijueque

Universidad Rey Juan Carlos, Madrid. España/Spain

sara.gallego@urjc.es

Recibido/Received: 14/05/2011

Aceptado/Accepted: 15/09/2011

\section{RESUMEN}

El objetivo de este trabajo es realizar un estudio sobre las teorías de redes sociales, y su importancia en la sociedad. Las redes sociales se han encargado durante todos estos siglos de preservar el valor comunitario, además de ser facilitadoras de interacción social en los procesos de desarrollo humano, gracias a la comunicación y relación entre individuos. Las redes sociales nos ayudan a entender el orden social, además proveen al ser humano de un espacio seguro y de apoyo, donde se integra y se adapta a los procesos y prácticas sociales.

\section{PALABRAS CLAVE}

Redes sociales, grupos primarios, sociedad civil, interacción, integración social, participación, acción social colectiva.

\section{SUMARIO}

1. Introducción. 2. Los grupos primarios como la génesis de las redes sociales. 3. Más allá de los grupos y las organizaciones: redes sociales. 4. Conclusiones. Bibliografía.

\section{ABSTRACT}

The aim of this work is a brief introduction to the concept of social networks and their importance in society. Social networks have been responsible over the centuries to preserve community values, in addition to being facilitators of social interaction in human development processes, through communication and relationships between individuals.

\section{KEYWORDS}

Networks social, primary groups, civil society, interaction, social integration, participation, collective social action.

\section{CONTENTS}

1. Introduction. 2. Primary groups as the genesis of social networks. 3. Beyond the groups and organizations: social networks. 4. Conclusions. References. 


\section{INTRODUCCIÓN}

Desde el comienzo de la humanidad el hombre ha necesitado un orden social (el cual se produjo gracias a la interacción entre individuos) para su persistencia o continuidad en el tiempo, este orden le ha proporcionado diferentes canales para el intercambio de información y diversas vías para el canje de prestaciones, bienes, etc. Este proceso de intercambio ha ido evolucionando a lo largo de los tiempos hacia una forma de estructura más compleja, de ahí la aparición de las redes sociales con el resultado principal: la continuidad de los grupos humanos.

Recordando la obra de Ernest Hemingway Por quién doblan las campanas, aparece un poema de John Donne, que dice así:

"Nadie es una isla, completo en sí mismo; cada hombre es un pedazo de continente, una parte de la tierra.; si el mar se lleva una porción de tierra, toda Europa queda disminuida, como si fuera un promontorio, o la casa de uno de tus amigos, o la tuya propia. La muerte de cualquier hombre me disminuye porque estoy ligado a la humanidad; por consiguiente nunca hagas preguntar por quién doblan las campanas: doblan por ti" (Hemingway, 1991:13).

Naturalmente, el individuo se encuentra ligado a la humanidad es algo indiscutible, además de ser una de la características principales del ser humano, ya que no podemos vivir en aislamiento, necesitamos de los demás para cimentarnos y completarnos. Gracias a esta unión o vinculación en colectivos, grupos o redes hemos sobrevivido a lo largo del tiempo superando todo tipo de advenimientos, desde catástrofes naturales, hasta las guerras más duras. Las redes nos han ayudado a reconstruir, lo que otras redes permitieron destruir.

Los seres humanos se sienten influenciados y afectados por los hechos y las acciones ya sean de carácter individual, o colectivo del grupo de personas más cercano a ellos, es decir, el grupo primario sin quitarle valor a la influencia del grupo secundario, y por supuesto teniendo en cuenta el entorno. Esta situación a la que se enfrenta todo individuo desde su origen, ha restringido, limitado y condicionado el comportamiento.

Los individuos tenemos la tendencia, por así decirlo, de imitar o copiar la conducta de aquellos con los que estamos en contacto directo. Sin duda son muchas cosas la que comunicamos y trasmitimos de una persona a otra, desde tristeza, generosidad, rabia, empatía, etc. De ahí que podamos deducir que las redes sociales, donde nos movemos, son las encargadas de determinar nuestros resultados dentro y fuera de ellas.

Otro de los factores que influye directamente en el comportamiento del ser humano es la manera en que se organiza y estructura la red. Los individuos, que componen determinadas redes o grupos donde conectan con personas que ya conocían, no son nada creativos ni obtienen los beneficios esperados, pasa lo mismo con los grupos formados por personas que se desconocen totalmente. En cambio en redes o grupos, donde algunas personas ya se conocían o existía algún tipo de contacto, y además se amplían con nuevos individuos, normalmente son muy creativos y obtienen beneficios.

Ahora bien, siendo conscientes de cómo afectan las redes sociales a los individuos, debemos esforzarnos en cambiar nuestro pensamiento hacia lo grupal y universal. Debemos de entender el mundo como un cosmos en red y nuestras reflexiones han de tender hacia un razonamiento en red. Es necesario dejar espacio y abrir nuestras mentes ya que la actitud de 
una sola persona no sólo afecta a su círculo más cercano como puede ser la familia, los amigos, sus vecinos, actualmente puede llegar y de hecho ya se ha producido, a toda la red social humana.

\section{LOS GRUPOS PRIMARIOS COMO LA GÉNESIS DE LAS REDES SOCIALES}

Para entender la naturaleza de las redes sociales, es necesario comenzar por el estudio de los grupos y su evolución. Podemos definir los grupos sociales "como una pluralidad de individuos que se hallan en contacto los unos con los otros, que tienen en cuenta la existencia los unos de los otros y que tienen conciencia de cierto elemento común de importancia. Los grupos por tanto son un nivel de agregación superior a la interacción, ya que implican una mayor pertenencia en el tiempo y una mayor densidad de las relaciones sociales implicadas. [...] La densidad de las relaciones sociales es variable pero suele ser mayor que la de una simple interacción" (Uña y Martín, 2009:122). De manera innata el individuo tiende a agruparse, independientemente de la forma que pueda llegar a adquirir la agrupación. Siempre existe un fin común a los miembros que pertenecen a dicho grupo, ya sea de carácter personal o impersonal. Los seres humanos tienen la necesidad vital de pertenecer a..., identificarse con... y tener un referente para autoevaluarse. Ello es necesario para que los seres humanos conserven su "Yo", y al mismo tiempo por ser miembros de un grupo sean conscientes de que existe un "Nosotros".

Una clave inestimable para aproximarse con una cierta seguridad al tema de las distintas formas de la sociabilidad humana "es la clásica distinción del alemán Ferdinand Tönnies entre comunidad (Gemeinschaft) y asociación (Gesellschaft). Esta distinción la aplica Tönnies a las relaciones sociales, a los grupos y a las sociedades, y ha influido profusamente en la tradición sociológica, desde Durkheim (sociedad de la solidaridad mecánica frente a sociedad de solidaridad orgánica) a Park (lo popular frente a los secular y Cooley (grupos primarios frente secundarios" (González-Anleo, 1991:133).

Definitivamente a lo largo del tiempo ha sido necesario generar una clasificación del grupo primario, secundario, comunidades, asociaciones, etc. El discernimiento seguido por los diferentes autores para diferenciar ambos grupos se localiza en el nivel de importancia particular que tiene el componente emocional de los miembros del grupo.

Siguiendo la línea de Cooley, respecto al concepto de grupos primarios y secundarios, él define el "grupo primario como un pequeño grupo social cuyos miembros comparten relaciones personales y duraderas Unidos por relaciones por relaciones primarias, por lo general, los individuos que pertenecen a un mismo grupo primario pasan juntos gran parte de su tiempo, ocupados en un conjunto amplio de actividades comunes y sienten que se conocen entre sí bastante bien [...] A diferencia del grupo primario, el grupo secundario es un grupo social grande e impersonal cuyos miembros persiguen un interés o actividad específica" (Macionis y Plummer, 2011:149). Nacemos dentro del seno de un grupo primario, su desaparición o destrucción puede afectarnos de una manera muy negativa a lo largo de nuestra vida, ya que en este tipo de grupos primarios se fragua gran parte de nuestra identidad y evolución a nivel individual y grupal/social. Las interacciones que se producen entre los miembros del grupo primario de alguna manera nos marcan para toda la vida, ya que son relaciones que involucran una gran carga emocional y afectiva, ello implica a su vez contacto físico directo, continuado en el tiempo, vivencias y experiencias compartidas de su existencia 
privada, empatía, interés desde la perspectiva de la preocupación por el estado del otro y si la situación lo requiere se presta ayuda económica y emocional de una manera incondicional, sentimiento de libertad, es decir, poder mostrar lo peor y lo mejor de sí mismo sin miedo al juicio y a las criticas. Se da una entrega personal de uno mismo al grupo, ya que el individuo no siente condicionamiento alguno por parte del grupo. Cada miembro del grupo primario percibe y siente que ocupa un lugar determinado que nadie más puede ocupar, de alguna manera siente que es imprescindible y vital en su grupo. Como es lógico para que se den este tipo de relaciones tan vitales para el ser humano el grupo primario nunca será muy extenso. Es difícil ingresar en grupos pequeños muy configurados, existen muchas restricciones como por ejemplo ideológicas. Todas estas características de los grupos primarios generan fuertes vínculos entre sus miembros que suelen durar toda la vida.

El ser humano no nace dentro de un grupo secundario, normalmente lo elige él o le eligen para que forme parte de dicho grupo. Las interacciones que se producen dentro del grupo secundario son de carácter contractual y funcional, por así decirlo, son relaciones poco profundas, frías y con un objetivo o meta determinado. Las emociones y los sentimientos se encuentran en un segundo plano, aunque en algunas ocasiones, con el tiempo y ante determinadas circunstancias, se puede establecer un acercamiento entre los individuos del grupo más emocional y vinculante. Son grupos más extensos y numerosos, de ahí que los vínculos que se generan no sean fuertes y estables, lo cual en parte dificulta que el acceso de nuevos miembros al grupo sea más frecuente y viable, los grupos secundarios son más abiertos, cambiantes, versátiles y volubles que los primarios. Los individuos que forman parte de los grupos secundarios no se muestran tal y como son, es decir, no muestran o intentan esconder sus carencias, defectos y debilidades, ellos mismo limitan y condicionan sus comportamientos frente al resto del grupo, ya que en este tipo de grupos existen juicios y críticas, además de normas y controles.

Ambos grupos, primarios y secundarios, son utilizados por los individuos como referentes para orientar y valorar (triunfos o fracasos), en definitiva, su propio comportamiento.

\section{MÁS ALLÁ DE LOS GRUPOS Y LAS ORGANIZACIONES: REDES SOCIALES.}

Las diferentes formas de agruparse en la sociedad han evolucionado a lo largo de la historia según las necesidades de los individuos. Desde las primeras comunidades o grupos primarios, que se generaron en el pasado, se han ido desarrollando otras formas de agrupamiento como las asociaciones o grupos secundarios, las organizaciones formales y, actualmente, lo que se conoce por redes sociales digitales. Las redes sociales van más allá de los grupos y las organizaciones. En los grupos y las organizaciones, por lo general, podemos identificar sus fronteras; esto es, podemos decir quiénes son sus miembros. Por el contrario, las redes son un campo de conexiones y relaciones: un conjunto de nodos (puntos clave) y un conjunto de lazos (o enlaces) que conectan algunos o todos estos nodos. Los nodos pueden ser personas, grupos, incluso países (Macionis y Plummer, 2011:165).

Félix Requena, en su libro Redes Sociales y Sociedad Civil afirma que "las redes inundan nuestro mundo. Actualmente el concepto de red es uno de los más poderosos en el análisis de la realidad social. De hecho, la realidad social se entiende mejor si la consideramos como un entramado de redes sociales: la estructura social es como una red. Pero, ¿cuál es la utilidad 
del análisis de redes en el estudio de la sociedad civil? El análisis de redes es una forma de comprender los procesos sociales" (Requena, 2008:1).

Efectivamente estudiar y conocer la evolución de las redes sociales es fundamental para comprender y esclarecer quiénes somos, en qué momento nos encontramos, y hacia dónde nos movemos. Para ello se realizará un recorrido de los diferentes enfoques y teorías: sociológica, psicológica y antropológica sobre el concepto de redes sociales.

Para Ligth y Keller, citados por Madariaga "las redes sociales son el tejido de relaciones entre un conjunto de personas que están unidas directa o indirectamente mediante varias comunicaciones y compromisos que pueden ser vistos como una apreciación voluntaria o espontánea, siendo heterogénea y a través de los cuales cada una de ellas está buscando dar y obtener recursos de otros" (Madariaga, Abello y Sierra, 2003:14). Ciertamente las redes sociales suelen aparecer de forma espontánea, sobre todo cuando el sistema empieza a quebrarse debido a las carencias y crisis económicas, políticas y sociales. En las redes sociales se dan relaciones de correlación que cubren necesidades.

El desarrollo del pensamiento sobre redes tuvo dos orígenes: "Primero surgió como un concepto sociológico al final de la década de los cuarenta, y sirvió para definir las interrelaciones entre un sistema social, como modelo alternativo para la entonces dominante perspectiva de acción estructural funcionalista. Desde este enfoque se hizo énfasis en las características de los lazos de unión entre la gente, a partir de las estructuras de las redes. El segundo parte de los desarrollos de la teoría de campo adaptada por Lewin, en la cual la conducta es vista como la función de una persona en una situación social. Esto significa que el entendimiento de la acción individual depende tanto del sistema social general en que se desarrollan las acciones como de las relaciones sociales de ese individuo con ese sistema" (Abello y Madariaga, 1999:18). En resumen la conducta del individuo está condicionada por la estructura o plataforma social, las circunstancias sociales que rodean al individuo y las interacciones que mantiene con otros individuos.

Respecto a la evolución de la red social y su uso, "las redes sociales son un conjunto de relaciones humanas que tiene un impacto duradero en la vida de cualquier persona. La red social está conformada por los sujetos significativos cercanos al individuo y constituye su ambiente social primario. Está constituida por los miembros de la familia nuclear, amigos, vecinos, compañeros de trabajo y conocidos de la comunidad [...]. Así mismo el uso de una red social desempeña varias funciones básicas para la supervivencia de sus miembros. Estas dependen de la clase de transferencia o intercambio que se produzca en la red. El uso de una red social busca la supervivencia para satisfacer necesidades básicas, desarrollo, que se utiliza para mejorar la calidad de vida de sus integrantes y también para buscar y mantener el vínculo social" (Abelló y Madariaga, 1999:19). Desde esta perspectiva es evidente que a través de las redes sociales el proceso de socialización se esparce y propaga, ya que estas redes nos sirven de plataforma o sostén para ello, proporcionándonos la capacidad de interactuar mediante la cual se produce la transferencia de valores, conocimientos etc., y el ambiente o entorno social adecuado cómodo y seguro.

Félix Requena coincide en la última parte de su definición de red social que se expone a continuación con Abello, a la hora de interpretar la red social como causante de la integración social. Requena define "la red social como un conjunto de puntos (actores sociales) vinculados por una serie de relaciones que cumplen determinadas propiedades [...]. La idea de red tal y como se quiere usar aquí, está tomada en gran parte de la teoría matemática de los grafos. En esta teoría se llama red a una serie de puntos vinculados por una 
serie de relaciones que cumplen determinadas propiedades. Es decir, un nudo de la red está vinculado con otro mediante una línea que presenta la dirección y el sentido del vínculo. [...] Entre dos puntos puede haber múltiples tipos de relación representadas por grafismos diferentes: estos multígrafos se utilizan cuando dos puntos están relacionados con más de un vinculo de naturaleza diferente [...] El concepto de red social también tiene un pleno sentido en su utilización como medio de integración social y de implicación de sujetos en el interior de un sistema social determinado; favoreciendo así el equilibrio dentro de un sistema social particular: ocupacional, político, etc.” (Requena Santos, 1989:137).

"Las redes sociales son un conjunto bien delimitado de actores-individuos, grupos, organizaciones, comunidades, sociedades globales, etc., vinculados unos a otros a través de una relación o un conjunto de relaciones sociales" (Lozares, 1996:109). Noción demasiado instrumental, ya que se deja de lado o se olvida de los sentimientos emocionales que afloran en las relaciones que se producen en determinadas redes sociales.

Elkaïm, (1987:12) afirma que una red social es un grupo de personas, miembros de una familia, vecinos, amigos y otras personas, capaces de aportar una ayuda y un apoyo tan reales como duraderos a un individuo o una familia. Es, en síntesis, un capullo alrededor de una unidad familiar que sirve de almohadilla entre esa unidad y la sociedad.

Montero al igual que Elkaïm, se detiene en el apoyo que prestan las redes sociales a los seres humanos, y además afirma que "la red es una forma de organización social en la cual se produce el intercambio continuo de ideas, servicios, objetos, modos de hacer. La red es sobre todo una estructura que permite difundir y detener, actuar y paralizar, en la cual las personas y la sociedad encuentran apoyo y refugio, además de recursos" (Montero, 2003:173). La autora hace referencia a la red como una organización social en donde la interacción entre los individuos funciona de una forma horizontal y transparente, donde la información, el conocimiento, los recursos e incluso la manera de crear, es compartida por todos los miembros, a diferencia de organizaciones e instituciones con estructura piramidal, donde las relaciones entre sus miembros son verticales, es decir, jerarquizadas y burocratizadas, el poder se concentra en una o varias personas debido a que son las que poseen la información, de ahí que sean también las encargadas de tomar decisiones por el resto de miembros que componen esa organización. Frente a estas organizaciones piramidales, en la línea de Montero, Madariaga, Abello y Sierra sostienen que "surgen otras formas de concebir la relación entre las personas: Las redes sociales. [...] las redes sociales son, entonces una opción que permite desestructurar los modelos piramidales, respetando características y potencialidades de las partes" (Madariaga, Abello y Sierra, 2003:11).

Montero, para corroborar su visión sobre el modelo organizacional de las redes sociales, el cual no es, ni debe de ser piramidal, cita a Itriago e Itriago para ellos "las redes son: un tejido vivo, integrado por comunidades, ergo seres humanos, cuya textura es activa, "llena de vida". Estos autores hacen referencia a la amplitud de su estructura, en la cual junto con las personas naturales, caben también las personas jurídicas, sin establecer jerarquías entre ellas, pues crean una estructura horizontal" (Montero, 2003:177).

Dabas también entiende la red social como un sistema abierto y donde las relaciones entre los individuos son horizontales, expone que la red social implica un proceso de construcción permanente tanto individual como colectivo. Es un sistema abierto, multicéntrico que, a través de un intercambio dinámico entre los integrantes de un colectivo (familia, equipo de trabajo, barrio, organización, tal como el hospital, la escuela, la asociación de profesionales, el centro comunitario, entre otros) y con integrantes de otros colectivos, posibilita la 
potencialización de los recursos que poseen y la creación de alternativas novedosas para la resolución de problemas o la satisfacción de necesidades. Cada miembro del colectivo se enriquece a través de las múltiples relaciones que cada uno de los otros desarrolla, optimizando los aprendizajes al ser éstos socialmente compartidos (Dabas, 1998:42). La autora entiende la red social como un sistema dinámico, por lo tanto cambiante y permutable, donde los individuos tienen la opción de poder entrar (lo que conlleva nuevas ideas y formas de hacer las cosas, en definitiva innovación) y salir, además de poder participar a través del intercambio aunque provengan de diferentes ámbitos. Para Dabas la red social, es un sistema que se retroalimenta a través de la reciprocidad de sus miembros, el cual se caracteriza por poseer un perfil más horizontal donde hay una distribución del poder, por así decirlo, en su organización y gestión.

Madariaga, Abello y Sierra coinciden con Dabas, a la hora de concebir el termino de red social. Ellos entienden que el "concepto de red social implica, entonces, un sistema abierto a través de un intercambio dinámico entre sus integrantes y con integrantes de otros grupos, que posibilita la potenciación de los recursos que se poseen y que se enriquecen con las múltiples relaciones entre los diferentes miembros que la componen. Las redes sociales son, en esencia, procesos dinámicos a través del tiempo y de las circunstancias sociales concretas" (Madariaga, Abello y Sierra, 2003:12).

Desde la misma visión que Dabas y Madariaga Abello y Sierra, Reales, Bohórquez y Rueda mantienen que "la red social puede ser conceptualizada como un sistema humano abierto, cooperativo y de propósito constructivo que a través de un intercambio dinámico (de energía, materia e información) entre sus integrantes permite la potencialización de los recursos que éstos poseen" (Reales, Bohórquez y Rueda, 1993:17).

\section{CONCLUSIONES}

Revisadas algunas definiciones sobre el concepto de redes sociales, se puede decir que las redes sociales son la plataforma básica donde se sostienen todas las interacciones individuales y colectivas de nuestras sociedades, edificadas por los seres humanos, y donde realmente existimos, coexistimos y funcionamos de una manera libre y autónoma. Las redes sociales son entes vivos que se nutren a través de las relaciones simétricas, recíprocas, flexibles y abiertas donde sus miembros pueden entrar y salir, cambiar de situación y función, lo cual es beneficioso para la innovación de esa red social, ya que siempre está recibiendo y reciclando ideas nuevas por parte de sus componentes. Las redes sociales engloban la heterogeneidad de individuos, grupos, organizaciones e instituciones, con intereses y objetivos comunes (en muchas ocasiones estos intereses pueden ser particulares, pero gracias al consenso y la negociación se busca lo mejor para todos) orientados hacia el bien común, lo que contribuye al desarrollo comunitario de la red, y ello a su vez incrementa el bienestar social y la calidad de vida. Donde se da una transparencia, visibilidad e intercambio de todos sus miembros en el manejo de la información, de ahí su dinamismo, retroalimentación y generación de conocimiento de forma colectiva. La red es la suma de la partes, todos los sujetos coparticipan y se involucran de la misma forma en la distribución, estructuración y organización flexible y consensuada, con el fin de lograr la meta común propuesta, ello genera ciertas responsabilidades y compromisos que se asumen por los involucrados. 
Gracias a las redes sociales el individuo cubre una necesidad básica para él que es la de convertirse en un ser social. Las redes sociales nos ayudan a entender el orden social, además proveen al ser humano de un espacio seguro y de apoyo, donde, a través de la relaciones con los demás, se integra y se adapta a los procesos y prácticas sociales, además de recibir el reconocimiento y la valoración social que es fundamental y necesaria para la homeostasis del ser humano. Este equilibrio individual se proyecta sobre la sociedad generando el equilibrio social colectivo. En el proceso de comunicación que se establece en las redes sociales de forma directa, se producen contactos cargados de emociones y sentimientos, o lo que es lo mismo el intercambio emocional, lo que permite la concepción de la confianza que es básica para el mantenimiento de vínculos y compromisos, lo cual es necesario e imprescindible para el afrontamiento de problemas, y lo más importante, el bienestar y funcionamiento individual y colectivo. Los individuos sienten que participan, ello genera en ellos un sentimiento de pertenencia que al unísono crea en ellos un sentimiento de responsabilidad y lealtad frente a su grupo y llegan a considerar a su grupo tan importante como ellos mismo, al mismo tiempo de sentirse integrados en su sociedad, ya que han construido una forma de cognición colectiva que es la encargada de fortalecer y reforzar la confianza y la reciprocidad de la red social o redes sociales a las que pertenecen. Gracias a las redes sociales, el proceso de socialización se difunde y se propaga, como podemos comprobar en lo anteriormente expuesto.

Las redes sociales son armas (herramientas) fundamentales de la masa humana, es decir, de la sociedad civil interpretada por Requena "como aquella que alude a las organizaciones, movimientos y asociaciones, así como a las relaciones entre ellas; es decir, a todo aquello que no es ni Estado ni mercado, pero que necesita de ellos para sobrevivir [...] es el conjunto de redes relacionales formadas para fortalecer a sus miembros. La sociedad civil se convierte de este modo en un espacio propicio para ejercer los principios de la ciudadanía democrática, es decir: la participación, [...] entendida como un conjunto interrelacionado de grupos intermedios entre el individuo y el Estado" (Requena Santos, 2008:33). Es a través de la sociedad civil donde se expresa toda la fuerza de la acción colectiva. Esta potencia colectiva es la encargada de producir movimientos y tendencias sociales, encargados de mover las potencialidades, recursos e intereses colectivos en beneficio de todos, de ahí que se pueda considerar a las redes como la columna social básica para los seres humanos.

Las redes sociales tienen su propio ritmo interno y externo, dependiendo del contexto, entorno y momento social, además de las circunstancias e historias de vida de sus integrantes.

En las redes sociales se invierte y se orienta toda la energía individual y colectiva de manera comprometida y responsable, con el fin de cambiar la trayectoria de las prácticas sociales y originar, nuevos efectos beneficiosos sobre la totalidad del colectivo. Estos efectos o resultados obtenidos se realizan a través de la participación que incluye todo tipo de aportaciones (proposiciones, soluciones, planes, opciones etc.) de todos los componentes a través de la acción, la teoría, y la administración y organización de las herramientas y recursos que poseen en la red. Debido a esta participación y colaboración por parte del individuo que compone una red social, se genera en él una actitud creativa y constructiva focalizada en el bien común y en el futuro colectivo, gracias a su trabajo y colaboración. Como observamos, las redes sociales producen la unión de la acción social individual para convertirla en acción social colectiva, la cual tiene más posibilidades de conseguir resultados óptimos, que si se realiza individualmente.

Para que realmente la génesis, construcción, evolución y funcionamiento de las redes sociales se pueda llevar a cabo de manera eficiente, tienen que cumplir con unos preceptos: 
En primer lugar, los intereses, metas y objetivos de las redes sociales han de ser comunes al colectivo, en ningún caso lucrativo y especulativo. En segundo lugar, las redes sociales deben encontrarse en situación de emancipación frente al Estado. En tercer lugar las redes sociales deben generar un ecosistema donde prime el respeto a la diversidad (ideológica, cultural, profesional, etc.), donde la participación sea consciente y general, la comunicación y el acceso a la información sea democrática, accesible, horizontal y cristalina. Abiertas y flexibles ante la entrada de nuevos miembros, interacciones con otras redes, y diversas circunstancias del contexto, es decir, tener la capacidad de encontrar soluciones ante determinados problemas de su entorno, para llevar a cabo nuevas y posibles adaptaciones a su medio social. Y, por último, que su existencia sea armónica con la comunidad.

\section{BIBLIOGRAFÍA}

ABELLO LLANOS, R. y MADARIAGA OROZCO, C. (1999): “Las redes sociales ¿Para qué?” en Psicología desde el Caribe. Barranquilla, Colombia, Universidad del Norte, $\mathrm{n}^{\circ}$ 2-3, pp. 119-125

DABAS, E. (1998): Redes sociales, familias y escuela, Buenos Aires, Paidós.

ELKAÏM, M. (1987): Les pratiques de réseaux: santé mentale et contexte social. Paris, E.S.F. GONZÁLEZ-ANLEO, J. (1991): Para comprender la sociología, Navarra, Verbo Divino.

HEMINGWAY, E. (1991): Por quién doblan las campanas, Chile, Andrés Bello.

LOZARES, C. (1996): "La teoría de redes sociales", en Revista de sociología, nº48, pp. 103-126

MACIONIS, J. y PLUMMER, K. (2011): Sociología, Madrid, Pearson Educación

MADARIAGA OROZCO, C., ABELLO LLANO, R. y SIERRA GARCÍA, O. (2003): Redes sociales: infancia, familia y comunidad, Barranquilla, Colombia, Universidad del Norte

MONTERO, M. (2003): Teoría y práctica de la Psicología Comunitaria. La tensión entre comunidad y sociedad, Buenos Aires, Paidós.

REALES, E., BOHÓRQUEZ, M. y RUEDA, A. (1993): Lineamientos conceptuales de las redes sociales. Una aproximación de la aplicación a la relación de la violencia. Bogotá, Universidad Colegio Mayor de Cundinamarca.

REQUENA SANTOS, F. (1989): "El concepto de red social", en REIS. Revista Española de Investigaciones sociológicas, Madrid. CIS, $\mathrm{n}^{\circ}$ 48/89, pp.137, 139,147.

REQUENA SANTOS, F. (2008): Redes sociales y sociedad civil, Madrid, CIS.

UÑA JUAREZ, O. y MARTÍN CABELLO, A. (2009): Introducción a la sociología, Madrid, Universitas.

\section{Breve currículo:}

\section{Sara Gallego Trijueque}

Licenciada en Sociología y Ciencias Políticas por la Universidad Pontificia de Salamanca, Máster en Psicología, Máster en Dirección y Gestión de Servicios Sociales, Máster en Sociedad de la Información y el Conocimiento, Curso de Altos Estudios Internacionales. Profesora ayudante en la UPSAM. Colaboradora de la AECID en las universidades de Pedagógica Experimental de Venezuela y en la Católica de Luanda (Angola). En Guatemala participó en el Programa de Autogestión de Educación. Actualmente es profesora lectora visitante en la URJC. Autora de diversos artículos en revistas científicas y congresos de Sociología nacionales e internacionales. Sus líneas de investigación se centran en la sociedad de la información y el conocimiento, redes sociales virtuales e identidad digital. 